\title{
Information, communication and computing technologies as enablers of advancements in modern information society
}

\author{
Anton $\mathrm{Kos}^{1} \cdot$ Yunchuan $\mathrm{Sun}^{2} \cdot$ Rongfang $\mathrm{Bie}^{3}$ \\ Published online: 25 March 2021 \\ (C) The Author(s), under exclusive licence to Springer-Verlag London Ltd., part of Springer Nature 2021
}

Information and communication technologies, along with advanced computing techniques and paradigms, are increasingly identified as enablers of new discoveries and advances in scientific fields such as education, finance, sports, social and human sciences, and others.

Recently, machine learning and deep learning methods, along with blockchain technology and the use of Big Data, have found a number of applications in finance, education, and social networking. For example, they are being used to extract knowledge from vast amounts of data, provide end users with recommendations for services and content, identify behavioral patterns, and help with preserving privacy.

The continuing miniatuarization of electronic devices, the availability and affordability of various sensors, and the ongoing development of the Internet of Things (IoT) have enabled a wide variety of solutions in sports, healthcare, and other areas that impact quality of life (QoL). Such examples include new smart devices and various sensor systems used in motor learning and performance improvement in sports, as well as motion/condition recognition in ubiquitous healthcare.

We expect further development and increased indisciplinary and transdisciplinary research in the above areas. This special

Anton Kos

anton.kos@fe.uni-lj.si

Yunchuan Sun

yunch@bnu.edu.cn

Rongfang Bie

rfbie@bnu.edu.cn

1 Faculty of Electrical Engineering, University of Ljubljana, Tržaška cesta 25, 1000 Ljubljana, Slovenia

2 Business School, Beijing Normal University, 19 Xinjiekouwai Street, Beijing 100875, China

3 School of Artifical Intelligence, Beijing Normal University, 19 Xinjiekouwai Street, Beijing 100875, China issue of Personal and Ubiquitous Computing is dedicated to the " Information, communication and computing technologies as enablers of advancements in modern information society " and presents a collection of papers that explore and address the challenges presented. Here, we introduce articles in the issue.

The first paper, "Event-based Summarization Method for Scientific Literature" explores the feasibility of systems for summarizing scientific literature that would provide brief and important dynamic information in which researchers are interested. Authors propose the automatic summarization based on the $5 \mathrm{~W} 1 \mathrm{H}$ event structure. Their experimental results show advantages in providing more detailed information, which is more convenient for researchers.

The paper "The role of technology for accelerated motor learning in sport" provides a brief introduction to motor learning in sport and its need for technology support. It also puts into context some technological trends and challenges in sport, such as the Internet of Things, Fog and cloud computing, smart sports equipment, and real-time biofeedback systems and applications.

The paper "Multi-object Tracking by Mutual Supervision of CNN and Particle Filter" proposes a Deep Learning based long-term tracking algorithm for traffic scenes. The experimental results show that the method can successfully track single and multi-objects in real-time for. The proposed algorithm can recover long-term tracking even in situations where the target disappears and reappears.

The paper "An active and dynamic credit reporting system for SMEs in China" presents an active and dynamic credit reporting framework based on Big Data and Blockchain for SMEs. It collects diversified data online, performs evaluation and analysis in real time, automatically generates online credit reports, and provides an effective way for various entities to interact. These functionalities are demonstrated through a case study from a real credit evaluation company.

The paper "Efficient continuous KNN join processing for real-time recommendation" proposes a locality-sensitive hashing (LSH)-based index called LSHI, which builds on 
the user set to efficiently find the specific users who might be affected by the updated content. The recommendation lists can be customized and the holistic effectiveness of the recommendation can be guaranteed. The advantages of the proposed method have been demonstrated through extensive experiments.

The paper "A novel distributed Social Internet of Things service recommendation scheme based on LSH forest" proposes a novel recommendation scheme called SIoT- SR, which uses LSH forest and a collaborative filtering algorithm to predict users' Quality of Service (QoS) data. It can achieve a good trade-off between storage space, accuracy, efficiency and privacy. The experimental results show that SIoT- SR has high prediction accuracy and efficiency while saving computational resources.

The paper "Multi-scale visualization based on sketch interaction for massive surveillance video data" proposes a multiscale approach that fuses on the data flow, objects and frames to achieve visualization of surveillance video data. The design of multi-scale structure makes the analysis of surveillance content natural and fluent with the annotation of video content. Its effectiveness has been proven through extensive user studies.

The paper "Cross-Device Task Interaction Framework Between the Smart Watch and the Smart Phone" introduces a framework for handling tasks between the smartphone and the smartwatch, including task delegation and task management. This framework can save valuable space on the phone screen for more complicated or important tasks. The user study shows that lightweight tasks can be handled efficiently by users on the watch.

The paper "An action identification method based on FSM and limb dry weight" investigates the motion recognition method used in the human-centered smart systems. The results, based on hierarchical probabilistic context-free grammar characterization of limb sequences using finite-state machine
(FSM), show that the proposed action recognition method applied to the same dataset has better recognition accuracy and lower time overhead than the other methods.

The paper "Utility Analysis on Privacy-Preservation Algorithms for Online Social Networks: An Empirical Study" uses various graph and application utility metrics to explore a tradeoff between privacy and data utility usability. The authors conducted an empirical study by implementing five state-of-the-art anonymization algorithms to analyze graph and application utility metrics on a Facebook and a Twitter dataset, and present interesting results.

The paper "Learning Behaviour Recognition Based on Multi-object Image in Single Viewpoint" proposes an innovative multi-model method to detect student learning behavior and count the number of students in classroom scenes. The proposed method outperforms other state-of-the-art methods on a real classroom surveillance dataset in terms of accuracy and speed.

The last paper, "Dynamic Resource Allocation Algorithm of Virtual Networks in Edge Computing Networks" proposes a dynamic algorithm for network resource requirement prediction based on the group search optimizer and the incremental design of the adial basis function network. Simulation results show good performance in terms of acceptance rate, network cost, link pressure and average revenue compared to traditional algorithms.

The Guest editors would like to thank authors for submitting the articles, reviewers for their constructive reviews that help improve the final version of manuscripts, and we would also like to express our honor to serve as the guest editors of this issue.

Publisher's note Springer Nature remains neutral with regard to jurisdictional claims in published maps and institutional affiliations. 